Original Article

\title{
A Survey To Evaluate The Nasolabial Angle (Nla) In Indian Population
}

\section{Vinaya Bhat ${ }^{1}$, Chethan Hegde ${ }^{2}$, Varun Raj ${ }^{3}$}

${ }^{1}$ Professor, ${ }^{2} \mathrm{HOD}$ and Professor, ${ }^{3}$ Post graduate, Department of Prosthodontics, A.B. Shetty M emorial Institute of Dental Sciences, $M$ angalore.

*Corresponding Author : Vinaya Bhat, Professor, Department of Prosthodontics, A.B.Shetty Memorial Institute of Dental Sciences, Mangalore. Mobile : +919481921180 E-mail : drvinayabhat@gmail.com

Received : : 18.01.2017 Abstract

Review Completed : 31.01.2017

Esthetics is an important factor in self-esteem of a person. Nasolabial angle is a key determinant

Accepted

: 05.02 .2017 in the fullness of the upper lip and it has been utilized to assess the esthetics while restoring upper anterior teeth. In the present study, the average nasolabial angle of the Indian population

Keywords : Esthetics, Nasolabial Angle (NLA), Edentulous patient, Complete Denture Prostheses, was assessed and identified which could be used as a guideline while replacing an edentulous patient with prosthesis.

Indian population.

\begin{tabular}{|c|}
\hline Access this article online \\
\hline Quick Response Code \\
\hline
\end{tabular}

\section{Introduction}

Physical appearance is an important characteristic of the face. It has long been established that self-esteem is strongly influenced by facial appearance. The perception of an attractive face is largely subjective, with ethnicity, age, gender, culture, and personality influencing average facial traits. Facial features have been evaluated forever by the researchers with the aim of contributing to esthetics. Various methods have been used to evaluate facial characteristics, such as anthropometry ${ }^{1}$, photogrammetry $2^{-4}$, computer imaging ${ }^{5}$, and cephalometry. ${ }^{6}$ Czarneckiet al. ${ }^{2}$ evaluated the perception of facial balance by varying the length of the nose, lip protrusion, and chin development. They found that the interrelationships of these facial features must be in balance in order to achieve racial harmony. The maxillary anterior teeth when present offer support to the upper lip giving the required fullness for esthetics. The positioning of the lip has been found to make an angle when measured with the base of the nose ${ }^{1}$. It has been referred to as Nasolabial Angle (NLA) and it is formed by 2 independent lines: one derived from the nose and another from the upper lip. On an average this angle has been identified as $90^{\circ}-110^{02}$. It represents an excellent clinical and cephalometric parameter to assess the anteroposterior position of the maxilla, in the treatment planning for patients with skeletal malocclusions, and particularly in the differential diagnosis of Class II malocclusions. An open or obtuse NLA suggests maxillary retrusion, and a closed or acute NLA suggests maxillary protrusion. $^{3}$

However, when an edentulous patient presents for prosthodontics replacement of the maxillary teeth, there is loss of this angle due to the collapse of the upper lip. 
Literature lacks any study done to assess the average NLA in Indian population. Hence, this study was undertaken to determine the average nasolabial angle to facilitate esthetic rehabilitation of Indian edentulous patients.

\section{Materials and Methods}

\section{A. Selection of participants}

The survey was conducted among undergraduate and postgraduate students of the A.B.ShettyM emorial Institute Of Dental Sciences after obtaining an ethical clearance and a consent from the participants.

The group consisted of 100 participants (60 males, 40 females)(Fig 04,05) aged between 20 and 30 years (mean age, 22.70 years) and with normal occlusion and facial harmony.

\section{Selection Criteria}

- Participants aged 20 - 30 years.

- Participants who did not undergo compensatory orthodontic or orthopedic treatment.

- Patient who did not undergo any type of facial cosmetic surgery,

- Participants with an oral prosthesis were excluded.

- Participants with malocclusion were also excluded.

- Patients with cleft lip and palate were excluded.

\section{B. Measuring NLA:}

4 protractors (Chamunda, Super) were utilized in this study to measure the nasolabial angle.

Each protractor was modified by cutting at angles $80^{\circ}, 85^{\circ}, 90^{\circ}, 95^{\circ}, 100^{\circ}, 105^{\circ}$ and $110^{\circ}$.

Thus, 7 individual modified protractors were made. (Fig 01)

The modified protractors were disinfected using $2 \%$ gluteraldehyde solution.

Participents were made to sit upright with his/her head straight and gazing at the horizon. The disinfected protractors were individually placed in such a way that the straight sides contacted the upper lipand base of the nose. The protractor which could be accommodated accurately was taken for measuring NLA for that participant. This procedure was carried out for all female (Fig 02) and male (Fig 03) participants. The value on the protractor was noted down for all the participants. The values were tabulated and average was calculated.(Table 1)

\section{Result}

The results showed that the average NLA in males was $99.67^{\circ}$ while in females it was $90.8^{\circ}$. The average NLA in Indian Population wasfound to be $95.23^{\circ}$.

\section{Discussion}

The present study was carried out to assess the average nasolabial angle in Indian population, so that these values can be used to restore the nasolabial angle in edentulous patients. In the population included in our study average NLA was found to be $95.23^{\circ}$.This result is in concurrence with the existing average value of NLA $\left(90^{\circ}-110^{\circ}\right)$ worldwide accepted and used

However, earlier studies have reported much higher NLA values $\left(114.08^{\circ} \pm 9.58^{\circ}{ }^{9} ; 102^{\circ 10} ; 105^{\circ} \pm 8^{011} ; 111.04^{08} ; 104^{\circ}\right.$ $\pm 11.5^{\circ}{ }^{12}$ and from $108.76^{\circ}$ to $114.40^{\circ}{ }^{13}$ ) than the values reported in our current study. This could be due to the difference in ethenic background of the population included in their study. Also, our group of participants consisted of younger population where the NLA could be smaller suggesting that this group presents a more biprotruded profile. ${ }^{8}$ Also in our study, the average nasolabial angle, among males were found to be $99.67^{\circ}$ and in females $90.8^{\circ}$,showing a difference in gender in the Indian population.

As can be observed in Table 1, the nasolabial angle in females was significantly smaller, confirming the existence of sexual dimorphism. This result differs from those of Silva Filhoet al. $^{13}$ and Fitzgerald et al. ${ }^{7}$, who found that individuals showed similar angular and linear measurements in both groups, male and female.

According to Prahl-Andersen et al. ${ }^{8}$ the tendency of the nasolabial angle is to decrease with age, specially until adolescence, when the growth of nose, chin and lips is expressed more intensely.Some studies have 
demonstrated that soft tissues that vary in thickness are the main factor in the determination of full facial profile.

In the present study, we evaluated the NLA in Indian population was found that the male participants had higher NLA than female participants. This may be taken to account as a guidance while restoring an Indian edentulous patient. Many authors have emphasized on soft tissue evaluation before contemplating orthodontic or orthognathic treatment modalities in which nasolabial soft tissue is an important factor in determining the patients' facial esthetics.

Fitzgerald et $\mathrm{al}^{7}$ proposed a consistent and reproducible method not only for constructing the nasolabial angle on a lateral cephalometric radiograph, but also to evaluate and develop standards for the inclination of the nose and the upper lip. The proposed method of locating the posterior

\section{References}

1. Maria B, Darcy F .Assessment of the nasolabial angle in young Brazilian black subjects with normal occiusion. Braz Oral Res.2004;18(3):233-7.

2. Czarnecki ST ,Nanda R S, Currier G F.Perceptions of a balanced facial profile.American Journal of Orthodontics and Dentofacial Orthopedics.1993;104:180-187.

3. Isiekwe GI, daCosta, IsiekweM C.Cephalometric assessment of the nasolabial angle of an adult Nigerian population. Nig Dent J. 2011;19,2:25-32.

4. Mayury K, Maria B etal.Nasolabial angle in finger sucking patients. BrazjOral Sci.2005;4:133-138

5. Daniel S, M arcos R, Guilher J, Karina M . Nasolabial Angle at Rest and Upon Smiling. JOralM axillofac Surg.2014; 72:2567.el-2567.e5.

6. Hasan A, Ghulam R .Evaluation of the nasolabial angle of Khyber pakhtunkhwa sample. Pakistan Oral \& Dental Journal.2014;34,1:106114.

7. Fitzgerald JP, Nanda RS, Currier GF .An evaluation of the nasolabial angle and the relative inclinations of the nose and upper lip. Am columella point onto which a tangent was drawn to the lower border of the nose, as well as the line from this point to labralesuperius proved to be a reliable technique for constructing the nasolabial angle.

\section{Conclusion}

Esthetics has been playing a major role in the success of many dental treatment. When there is loss of references as in edentulousness, to restore the patient to his/her original esthetics is a herculian task. Nasolabial Angle (NLA) which is one of the factors in esthetics is an important reference that a prosthodontist can rely on while restoring an edentulous patient. In our study of Indian population, it was found to be within the average $\left(90-110^{\circ}\right)$ which has been applied in use universally. This factor may be applied as an index while restoring upper lip fullness during Contouring of the maxillary occlusion rim.

Jorthod. 1992;102:328-34

8. Prahl-Andersen etal.Adolescent growth changes in soft tissue profile. American Journal of Orthodontics and Dentofacial Orthopedics; 1995:107:476-483.

9. Nandini et al .An evaluation of nasolabial angle and the relative inclinations of the nose and upper lip. Jcontemporery dental practice;2011;12(3):96-105.

10. Elias AC.The importance of the nasolabial angle in the diagnosis and treatment of malocclusion. IntJ Orthod 1980;18:7-12.

11. Cotton WN, Takano WS, Wong WM W.The Downs analysis applied to three other ethnic groups. Angle Orthod 1951;21:213-20.

12. BurstoneCJ. lip posture and its significance in treatment planning..Am Jorthod.1967;53:262-84

13. Silva $F$ et al.Avaliac;aoce- falometrica do angulonasolabialaos 7 anos, 12 anos e 19 anos, numaamostra de oclusaonormal.Rev SB01990;1:108-13. 